\title{
Image Processing System for Ultrasound Images of the Eye
}

\author{
M. Lepine Shaw, H. Hannoon, R. Youmaran, A. Adler \\ Department of Systems and Computer Engineering \\ Carleton University,Ontario,Canada adler@sce.carleton.ca
}

\begin{abstract}
Closed Angle Glaucoma causes an increase in fluid pressure in the eye due to decreased fluid flow between the iris and the cornea. To diagnose Glaucoma, ultrasound images of the eye are taken and a technician studies the images and takes measurements. This process is time consuming, and results vary among technicians. In a previous paper, we developed an image processing algorithm to extract several key landmark features in the iris. In this paper, we extend this algorithm to calculate the complete set of diagnostic parameters from ultrasound biomicroscopy. A robust algorithm is described to calculate the parameters which describe the thickness of iris; ID1, ID2, and ID3. The method is tested on 89 clinical images and compared to the values identified by the technician. Results indicate good agreement in $97 \%$ of cases.
\end{abstract}

\section{INTRODUCTION}

Closed Angle Glaucoma causes an increase in fluid pressure in the eye due to a decrease in fluid flow between the iris and the cornea. One accurate technique to monitor the progression of glaucoma and the patient's response to medication is using ultrasound biomicrosopy; high-frequency ultrasound images of the iris are taken. Images are currently evaluated by a technician to measure diagnostic parameters. This process is time consuming, and, results can vary depending on the technician [3]. In order to save diagnostic time and increase repeatability, we developed an image processing algorithm to extract the key image landmarks: the scleral spur, and apex point [1]. This paper extends this work to calculate the complete set of diagnostic parameters from ultrasound biomicroscopy. Specifically, the iris thickness parameters (ID1, ID2, and ID3) are computed in a robust way (see fig. 1). These parameters are defined as follows [2]: ID1 is on a line perpendicular to the iris connecting a point on the trabecular meshwork, $500 \mu \mathrm{m}$ from the scleral spur, to the ciliary process. ID2 is perpendicular to the iris and $2 \mathrm{~mm}$ from scleral spur. ID3 is the thickest part of the iris near the lens iris apex.

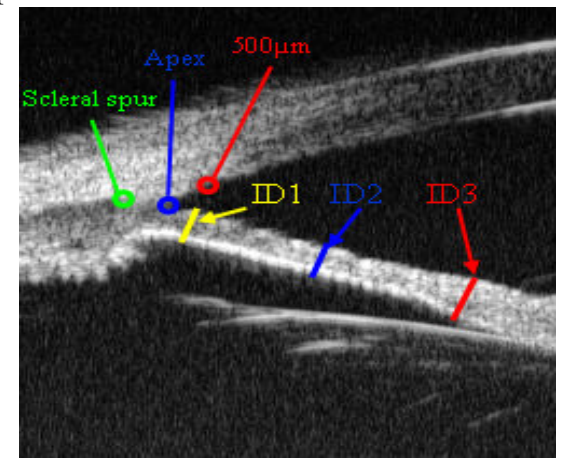

Figure 1: An ultrasound image of the eye and the parameters, scleral spur, apex, $500 \mu \mathrm{m}, \mathrm{ID} 1, \mathrm{ID} 2$, and ID3.

\section{ALGORITHM DESIGN}

This section describes the implemented algorithm. The algorithm depends on three parameters from our previous result [1]: the scleral spur, the apex point, and the point situated at $500 \mu \mathrm{m}$ from the scleral spur. One of the key challenges in automatically processing biomedical ultrasound images is the large level of speckle noise. The overall flowchart of the proposed algorithm is shown in figure 2. The algorithm consists of a main part to isolate the iris region and of three subparts to measure each parameter.

\section{A. Isolate Iris algorithm}

The parameters chosen are all on the iris, therefore isolating the iris enables measuring of the parameters without interference from other structures, such as the cornea and lens. The iris is isolated by cropping the image at the apex point and selecting the object with lowest centroid as the iris. In some cases the lens is attached to the iris and must be removed. Removing the lens is done in a way similar to isolating the iris, and requires finding the iris-lens apex point which is useful in measuring ID3. The Isolation iris algorithm is shown fig. 2, blocks 1.1 to 2.4 .

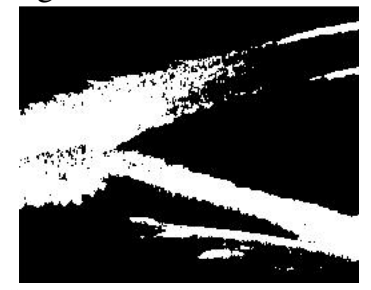

Figure 3: after thresholding.

1.1 Thresholding: The threshold is chosen by applying the Gray threshold algorithm to the image (fig. 3).

1.2. Hole filling: Small openings in thresholded image, $\mathrm{f}_{\mathrm{th}}$, are found and filled as seen in figure $4 \mathrm{a}$.

1.3. Region classification and segmentation for the eye: All of the closed objects in $\mathrm{f}_{\text {fill }}$ are examined. The object with the largest area is selected as the eye. All other objects in $\mathrm{f}_{\text {fill }}$ are set to 0 , leaving just the eye. The resulting image is $\mathrm{f}_{\text {eye }}$.

1.4. Vertical cropping: The image of the eye, $\mathrm{f}_{\mathrm{eye}}$, is cropped between the Apex $x$, plus an offset, and the end of the image, isolating the right side of the cornea and iris (fig. 4a). The offset is added to ensure the cornea and iris are not connected. 


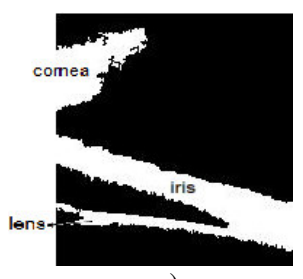

a)

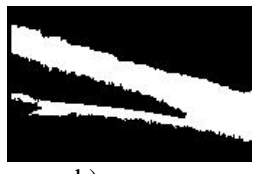

b)

centroid of the objects. The two largest objects are classified as either being the cornea or the iris. Then the object with lowest centroid is classified as being the iris.

Since the lens may be attached to iris and will therefore have to be isolated and removed. To determine if the lens is attached, the area between the iris and cornea is found. If no area is found, then it is assumed that the lens was not attached and the isolate iris algorithm is complete; if found, the iris-lens apex is found and used to isolate the lens.

Figure 4: a) The result of hole filling and cropping the thresholded image. b) The iris and lens with the cornea removed.

1.5. Region classification and iris segmentation: All closed objects in $\mathrm{f}_{\text {crop } 1}$ are analysed to identify the iris, seen in figure $4 \mathrm{~b}$. Classification is done according the area and

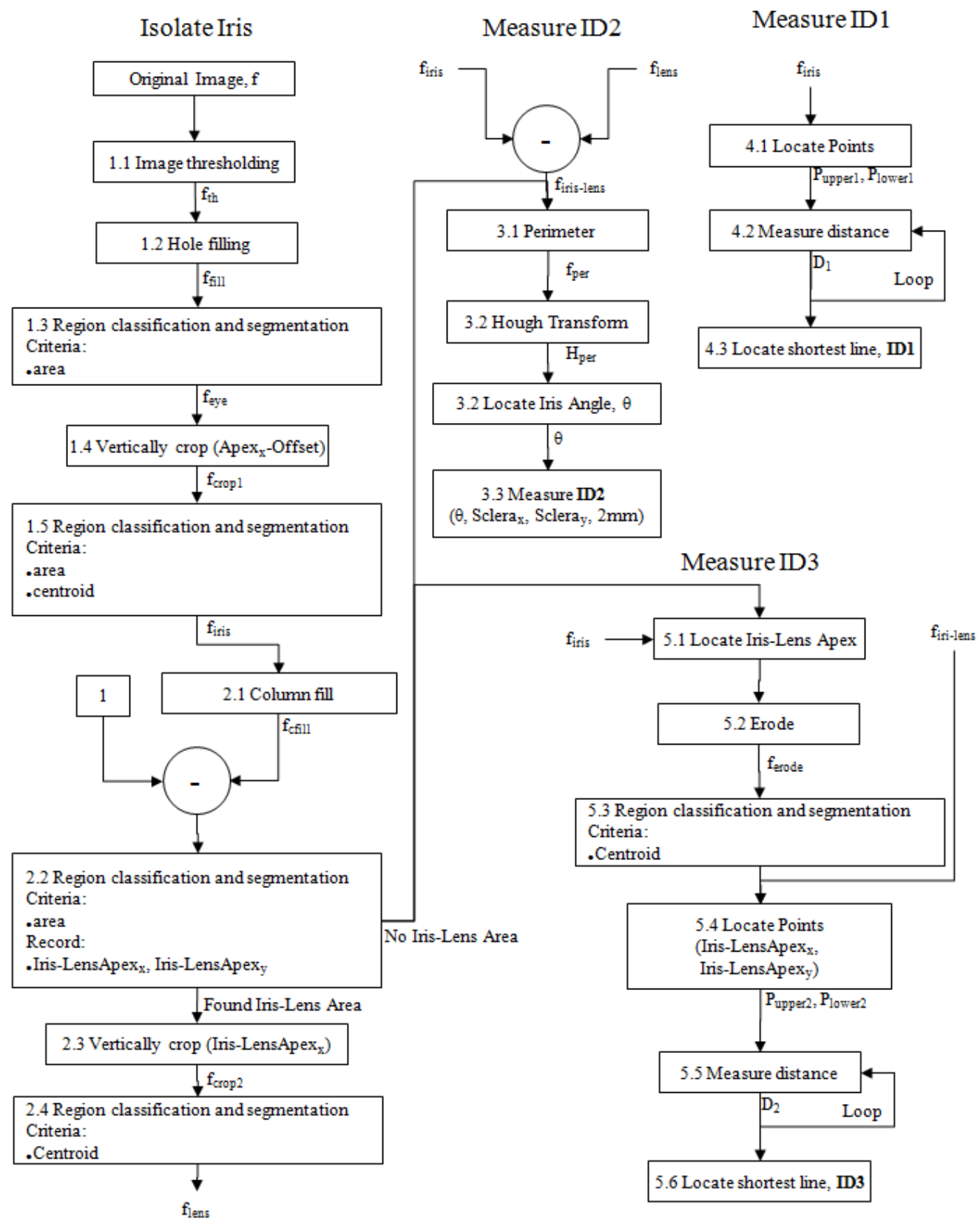

Figure 2: A flow chart describing the algorithm used to isolate the iris of an ultrasound image of the eye, and to measure the parameters ID1, ID2, and ID3.

The following describes the steps for isolating the lens as in the flowchart, figure 2.

2.1 Column fill: In order to find the area between the iris and the lens, a column fill operation is applied to the image $f_{\text {fill. }}$. The column fill operation sets pixels with the value 0 to 1 in the columns of the image starting from the bottom until it encounters a pixel set to 1 . After, the remaining unfilled area above the iris is filled using a flood fill operation. This 
effectively isolates the areas in between objects which are vertically aligned, such as the area between the iris and lens, seen in figure 5 . The resulting image is $\mathrm{f}_{\text {cfill }}$.

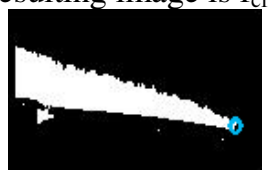

Figure 5: The isolated iris and lens with the column fill operation applied. The white area represents the area in between the iris and the lens. The blue dot represents the iris lens apex.

\subsection{Region classification and segmentation of iris lens:} The objects' area in $\mathrm{f}_{\text {cfill }}$ is used as the criteria for finding the lens. Objects smaller then a threshold were ignored, and the object with the greatest area is classified as being the area in between the iris and lens, seen in figure 5. If no object is found which meets the criteria, it is assumed that the lens was not attached to the iris, and $f_{\text {iris }}$ is used as the image for measuring the parameters and algorithm jumps to 3.1 in the flowchart, figure 2 . If the area between the iris and the lens is found, the iris-lens apex is recorded as the right most pixel of the area classified as in between the iris and lens (fig 5).

2.3 Vertical crop: The image of the iris and lens, $\mathrm{f}_{\text {iris }}$ is cropped between the start of the image and the iris-lens apex, in order to separate the lens and the iris (fig 6a).

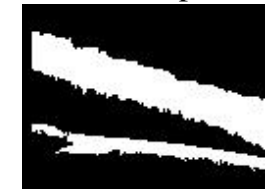

a)

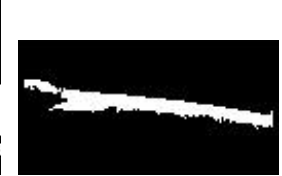

b)

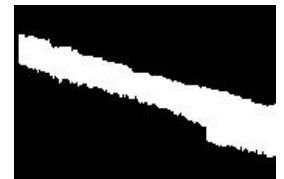

c)
Figure 6: a) The result of cropping at the iris-lens apex point. b) The isolated lens. c) The iris with the lens removed.

2.4 Region classification and segmentation of the lens: The lens is classified as the object with the lowest centroid in the image $f_{\text {crop2 }}$. The result is an image of the lens, $f_{\text {lens. }}$. With the lens isolated, $\mathrm{f}_{\text {lens }}$, (fig. $6 \mathrm{~b}$ ) it can be subtracted from the iris, $\mathrm{f}_{\text {iris. }}$ The resulting image is $\mathrm{f}_{\text {lens-iris }}$, (fig 6c.)

\section{B. Measure ID2}

As described, ID2 is the length of a line $2 \mathrm{~mm}$ from the scleral spur and perpendicular to the iris. The following describes how ID2 is measured in the flow chart (fig.e 2).

3.1 Perimeter: The perimeter operation is applied to $\mathrm{f}_{\text {lens- }}$ iris if the lens was found; otherwise it is applied to $f_{\text {iris. }}$. The purpose of this is to simplify the Hough transform operation applied in the next step. The resulting image is $\mathrm{f}_{\text {per }}$.

3.2 Hough Transform: The Hough transform is applied to $f_{\text {per }}$ to determine the angle of the iris relative to the image space. The Hough transform re-paramatizes each pixel in an image to the form:

$$
r(\theta)=x_{o} \cos (\theta)+y_{o} \sin (\theta)
$$

where $\mathrm{x}_{0}$ and $\mathrm{y}_{0}$ represent image pixel coordinates. The result of the Hough transform applied to $f_{\text {per }}$ is the accumulator matrix $\mathrm{H}_{\text {per. }}$.

3.3 Locate Iris Angle, $\theta_{\text {iris }}$ : The angle of the iris, $\theta_{\text {iris }}$ is located by selecting the element in the accumulator matrix with the greatest value. Elements for angles between 80 and
100 degrees are ignored as often they represent the lines that are ends of the iris.

3.4 Measure ID2: Pixels which lie on the line $2 \mathrm{~mm}$ from the scleral spur and rotated 90 degrees plus the iris angle are selected and measured as ID2 (fig 7).

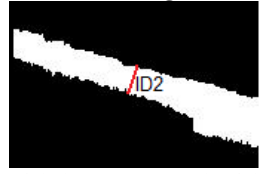

Figure 7: The isolated iris with lens removed with ID2 in red.

\section{Measure IDI}

The thickness of the iris along the line trabecular ciliary process distance (TCPD) is denoted by ID1. The line TCPD is a perpendicular line from the point located $500 \mu \mathrm{m}$ away from the scleral spur through the iris to the ciliary process (fig 1). The algorithm is 4.1 to 4.3 in the flowchart (fig. 2).

4.1 Locate Points: From the apex point and the point located $500 \mu \mathrm{m}$ away from the scleral spur, we iterate through pixels in the columns above the iris to determine $P_{\text {upper }}$. Similarly, the $P_{\text {lower }}$ points are determined by iterating over column pixels (fig. 8).

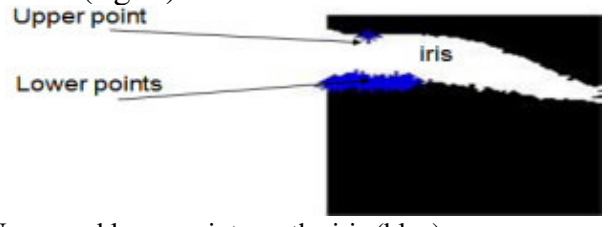

Figure 8: Upper and lower points on the iris (blue)

4.2 Measure distance: The measured distances (d) between the upper and the lower points are determined by the Euclidean distance from $\left(\mathrm{x}_{1}, \mathrm{y}_{1}\right)$ to $\left(\mathrm{x}_{2}, \mathrm{y}_{2}\right)$, where $(\mathrm{x}, \mathrm{y})$ represents the (row,column) pixel locations. This step is repeated for each lower points. Each measurement is saved in a variable (fig. 9).

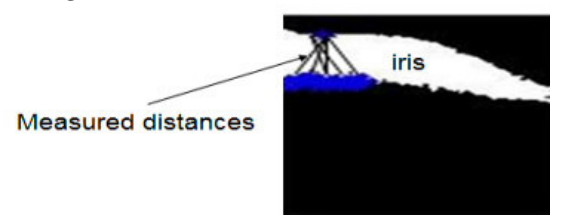

Figure 9: Measuring the distances between the points

4.3 Locate shortest line: The measured distances are compared to determine the shortest. This value is converted to $\mu \mathrm{m}$ units and identified as ID1 (fig 10).

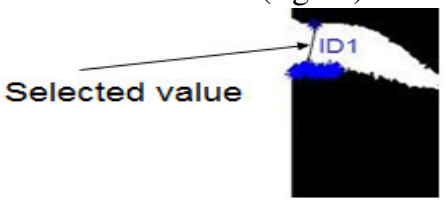

Figure 10: Extracted ID1 as the shortest distance

\section{Measure ID3}

ID3 represents the thickness of the iris at the right margin of fig 1. This step is represented in blocks 5.1 to 5.6.

5.1 Locate Iris-Lens Apex: The iris-lens apex point is where the iris and the lens join, in $\mathrm{f}_{\text {iris }}$. In some cases ID3 is located perpendicularly on the iris at the apex point. Other cases (when the lens does not exist) ID3 is the thickness of the iris at the edge. The apex point is determined by iterating 
through the pixels in the rows below the iris and finding the farthest point on the iris (fig. 11).

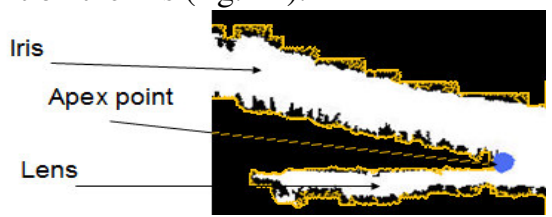

Figure 11: Determining the apex point

5.2 Erode: As shown in figure 12-(a), in some cases the end of the iris is attached to the lens. In these cases, it is necessary to erode the image and separate the iris from any other parts attached to it (fig 12-(b)).

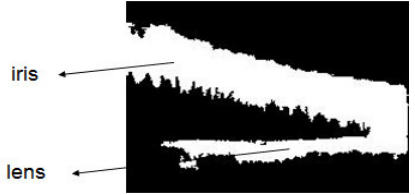

a)

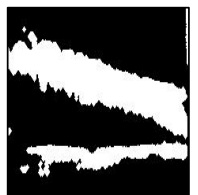

b)
Figure 12 a) Iris and lens attached. b) Eroded image

5.3 Region classification and segmentation: Each object in the image $\mathrm{f}_{\text {erode }}$ is identified and classified. The largest two objects are the iris and the lens. The location of the iris is higher than the location of the lens; thus, the iris and the lens are identified based on the centroid of each object (fig 13-(a) and 13-(b)).

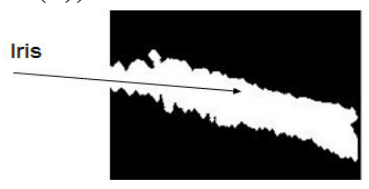

a)

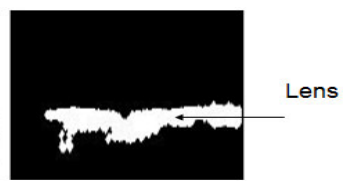

b)
Figure 13 a) Extracted iris b) Extracted lens

5.4 Locate Points: Based on the apex point, found before, the upper points at the end of the iris are determined by iterating through the pixels in the columns above the iris as seen in figure 14 .

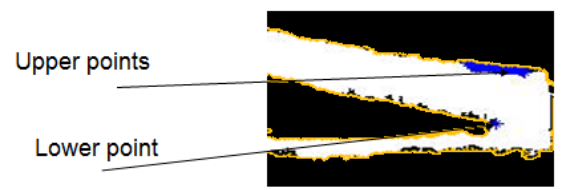

Figure 14: Determining the upper and the lower points on the iris.

5.5 Measure distance: The distances between the upper points and the lower point which are located at the end of the iris are determined by calculating the Euclidian distance. This step will repeat for each of the upper points. As shown in figure 15, different distances will be calculated and stored.

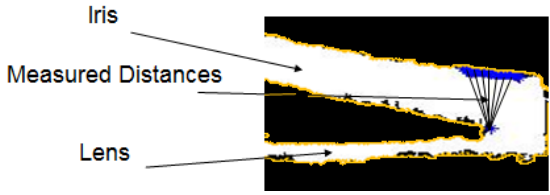

Figure 15: Different measured distances between the points

5.6 Locate shortest line: The measured distances are compared with each other and the shortest line is extracted as being perpendicular to the iris. The selected distance value is converted from pixels to meters. In doing so, ID3 is determined as seen in figure 16.

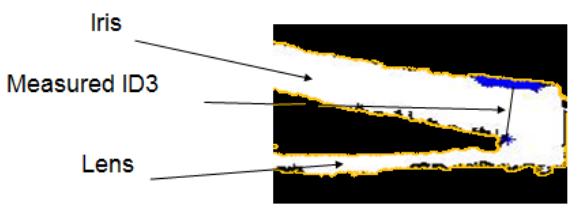

Figure 16: Measured ID3, the shortest distance

\section{RESULTS}

Eighty-nine images were obtained from patients at the University of Ottawa Eye Institute using the Ultrasound Biomicroscope (UBM) System Model 840 (ZeissHumphrey), using the protocol described in [3]. The images were delivered in PCX format and were 256 by 256 pixels representing $5 \mathrm{~mm}$ by $5 \mathrm{~mm}$ with a grey scale depth of 256. The proposed algorithm was implemented in Matlab R2007a. The isolate iris algorithm was tested by comparing binary images of manually isolated irises to the binary images produced by the algorithm. The images were compared by applying an Exclusive OR to two corresponding images. If the difference was more than $40 \%$ of the area of the iris, it was determined that the algorithm failed to isolate the iris. The threshold value was chosen to be $40 \%$; this value was determined to be small enough to detect the cases when the cornea had failed to be removed. The algorithm successfully isolated the iris for 86 of the 89 images. The 3 failures were well over the threshold and occurred when the algorithm selected the cornea instead of the iris. Next the 3 clinical parameters were manually measured as described in [2] for each of the 86 images which passed the iris isolation test and then compared to the algorithms results. It was found that there was an average percent difference of $6.9 \%, 8.7 \%$, and $9.2 \%$, for ID1, ID2, and ID3 respectively between the algorithm's results and the manually measured images.

\section{DISCUSION}

This image processing system implements an algorithm to isolate and measure the thickness of the iris. It would be of interest in a dose-response study to assist patients at risk of Glaucoma. This system isolates the iris successfully and showed a percent difference from manually measured images of $6.9 \%, 8.7 \%$, and $9.2 \%$, for ID1, ID2, and ID3 respectively. Since results can be easily superimposed on the images, they can be verified, and failures are visible.

\section{REFERENCES}

[1] R.Youmaran, P.Dicorato, R.Munger, T.Hall, A.Adler, "Automatic Detection of Features in Ultrasound Images of the Eye", IEEE Conf Instrum Meas, pp. 1829-1834, Ottawa, Canada, 17-19 May, 2005

[2] Pavlin CJ, Harasiewicz K, Foster FS, "Ultrasound biomicroscopy of anterior segment Structure in normal and glaucomatous eyes", Am J Ophthalmology, 113:381-9, 1992

[3] Daneshvar H., Brownstein S., Mintsioulis G., Chialant D., Punja K. Damji KF., "Epithelial ingrowth following penetrating keratoplasty:A Clinical, Ultrasound Biomicroscopic and Histopathological Correlation", Can J Ophthalmology, 35:222-224, 2000 